Nos adresses postales pour les envois:

Julien Brachet

UMR 201 "Développement \& Sociétés"

Université Paris 1 Panthéon-Sorbonne - IRD

45 bis, Avenue de la Belle Gabrielle

94736 Nogent-sur-Marne

Julien.Brachet@ird.fr

Armelle Choplin

Université Paris-Est - Marne-la-Vallée

UFR SHS, Bois de l'Etang,

Cité Descartes - 5 boulevard Descartes - Champs-sur-Marne

77454 Marne-la-Vallée cedex 2

armelle.choplin@univ-paris-est.fr

Olivier Pliez

Laboratoire LISST, UMR 5193

Maison de la recherche

Université Toulouse le Mirail

5 allées Antonio Machado31058 Toulouse cedex 9

pliez@univ-tlse2.fr

Une (1) carte, intitulée "Itinéraires des migrations (trans-)sahariennes 》, située à la fin de l'introduction

\title{
LE SAHARA ENTRE ESPACE DE CIRCULATION ET FRONTIÈRE MIGRATOIRE DE L'EUROPE
}

Julien Brachet, chargé de recherche à l'IRD, UMR Développement \& Sociétés (IRD - Université Paris 1).

Armelle Choplin, maître de conférences en géographie, Université Paris-Est Marne-la-Vallée, EA 3350 Analyse Comparée des Pouvoirs / UMR 8586 PRODIG.

Olivier Pliez, géographe, chargé de recherche au CNRS, LISST (UMR 5193, CNRS-EHESS-UTM).

\section{Résumé}

Les migrations internationales au Sahara représentent aujourd'hui un enjeu central des relations géopolitiques entre les États d'Afrique subsaharienne, d'Afrique du Nord et d'Europe. Elles ne constituent pas un phénomène nouveau puisque dès le milieu du $\mathrm{XX}^{\mathrm{e}}$ siècle des ressortissants des États sahéliens se rendaient au Maghreb pour y travailler , ces mobilités se réalisant en étroite relation avec la mise en valeur du Sahara. Pourtant, depuis le début des années 2000, la médiatisation des migrations à destination de l'Europe, très minoritaires au regard de l'ensemble des flux, a radicalement changé la donne. Chaque migrant de la région est suspecté d'être un "clandestin en transit vers l'Europe". Les politiques migratoires depuis lors mises en oeuvre dans les régions sahariennes perturbent l'ensemble des mobilités dans cet espace en passe de devenir une nouvelle frontière migratoire de l'Europe.

Abstract The Sahara: internal mobility and the southward extension of European borders. International migrations in the Sahara are one of the most important issue of the relations between 
sub-Saharan Africa, North Africa and Europe. They are not a new phenomenon. Since the 1950s, migrants from the Sahel have travelled to the Maghreb for work, which was plentiful as North African governments invested in their Saharan territories. Since the early 2000s, media coverage of migrations through the Sahara to Europe - that constitute but a minute fraction of overall Saharan migrations - has perturbed these longstanding patterns of mobility. Now, each sub-Saharan migrant in the Sahara is suspected to be in transit to Europe, and North Africa as a whole is re-defined, through the imposition of European migration policies, as the EU's southernmost border.

\section{Introduction}

Les migrations internationales entre les deux rives du Sahara, fortement médiatisées ces dernières années, sont dorénavant au cœur des relations diplomatiques entre l'Europe, l'Afrique du Nord et l'Afrique subsaharienne. Dans cette zone, plus un accord de coopération avec l'Union européenne ne se fait sans une clause sur la migration, plus un sommet réunissant des ministres ou des chefs d'États des deux continents ne se termine sans qu'ait été abordée la «gestion » des circulations migratoires, plus une crise politique ne se passe sans être observée à travers le prisme des migrations irrégulières potentielles. Dans l'effervescence de cette actualité et face aux enjeux géopolitiques et électoraux qui lui sont associés, les migrations au Sahara ont bien souvent été perçues et représentées de manières simplificatrices voire partisanes, à travers des focales immodérément européano-centrées. Tout migrant originaire d'Afrique subsaharienne se retrouvant aujourd'hui au Nord Sahel ou au Sahara n'est-il pas en effet considéré, peu ou prou, comme un potentiel candidat à l'émigration dite «clandestine» vers l'Espagne ou l'Italie ? Cette vision, partagée par de nombreux acteurs institutionnels, s'inscrit dans une lecture géopolitique du monde marquée depuis le début des années 2000 par la recatégorisation des espaces au prisme des considérations sécuritaires. Aussi erronée soient-elles, ces représentations émaillent les propos de maints dirigeants européens et nord-africains qui se livrent sans complexe à des amalgames ou des outrances verbales. Umberto Bossi, membre du gouvernement italien, entendait par exemple dès 2003 "faire tonner le canon» pour empêcher les bateaux transportant des migrants irréguliers d'approcher des rives italiennes ${ }^{1}$. Plus récemment, en mars 2011, la député UMP Chantal Brunel suggérait de remettre les immigrés «dans les bateaux » afin de "rassurer les Français ». Derrière ces propos xénophobes se lisent en filigrane des volontés de plus en plus explicites d'une remise en cause du droit maritime, du droit d'asile et plus globalement des droits de l'homme, permettant un traitement d'exception extrêmement violent et parfois criminel des migrants africains en route vers l'Europe, ou simplement supposés l'être. De l'autre côté de la Méditerranée, les dirigeants jouent également de la peur européenne de l'invasion pour utiliser ces mêmes migrants comme objets de marchandage. Afin de justifier le renforcement du contrôle aux frontières, le ministre mauritanien de la défense déclarait en 2010 que son pays était «sur le chemin de l'immigration clandestine, de la drogue et du trafic d'armes $»^{2}$. Quant à Mouammar Kadhafi, quelques mois seulement avant d'être à nouveau érigé par les Européens en figure de l'ennemi-dictateur, il annonçait aux côtés de Silvio Berlusconi à Rome que "pour "stopper" l'immigration clandestine [...], la Libye soutenue par l'Italie demande à l'Union européenne au moins 5 milliards d'euros par an $\rangle^{3}$.

Ce primat du point de vue européen et sécuritaire se traduit depuis plusieurs années par l'idée que l'externalisation du contrôle au-delà des frontières de l'Europe constitue le moyen le plus efficace pour mettre un terme aux arrivées de migrants africains en situation irrégulière au cœur d'une Europe qui se voudrait forteresse. De ce fait, des villes aux grands ergs, les espaces sahariens traversés sont labellisés de « transit » afin de justifier leur contrôle. Les imaginaires des grands

\footnotetext{
$1 \quad$ Le Monde, 29 juin 2003.

$2 \quad$ Le Monde, 4 juin 2010.

3 AFP, 31 août 2010.
} 
espaces désertiques se renouvellent en effet sans cesse, au risque de la généralisation abusive, d'un moindre souci du détail et de raccourcis quant à la réalité vécue par ceux qui y circulent. Achille Mbembe [1999, 2000] étayait ainsi à la fin des années 1990 le constat d'une «déterritorialisation... sur les pourtours du désert du Sahara», évoquant un désert "parcouru par des acteurs tant étatiques que non étatiques, par des nomades, des commerçants et des aventuriers [où] la forme de territorialité dominante est itinérante et nomadique ». On retrouve paradoxalement dans les propos d'un des tenants de la pensée postcoloniale, tout un outillage de la pensée, déjà datée, d'un Sahara mystérieux, incontrôlé et dangereux. Ce genre de représentations permet ensuite d'admettre plus aisément les fatalités de la géographie saharienne souvent mobilisées dans les rapports des missions techniques, telle que celle effectuée sur l'immigration illégale en Libye (4 avril 2005) à la demande du Conseil de l'Union européenne. Il y était par exemple écrit que " le contrôle des frontières [de la Libye] est significativement affecté par leur longueur (4 400 kilomètres de frontières perméables avec six pays, et une ligne de côte de 1770 kilomètres) ». C'est indéniable mais c'est aussi négliger l'importance des routes et des villes qui trament cet espace migratoire. Dans le même temps, les représentations cartographiques d'un Sahara considéré comme un espace migratoire « lisse », c'està-dire où le trait de dessin continu de quelques routes migratoires occulte toutes les « aspérités » spatiales, politiques, temporelles, pécuniaires, ...- qui jalonnent les itinéraires empruntés par les migrants ${ }^{4}$, ont aussi abondé. Elles servent d'arguments aux stratèges de la géopolitique régionale tentant de dessiner une " géographie globale des risques et des menaces ».

S'intéresser aux migrations dans l'espace saharien nécessite pourtant de distinguer plusieurs échelles spatiales et temporelles afin d'aller au-delà de "l'événement migratoire » que constituent les flux à destination de l'Europe. Ces derniers à la fois relativement récents -depuis le début des années 1990- et marginaux d'un point de vue quantitatif, sont déjà largement remis en cause par la collaboration entre pays riverains du nord et du sud de la Méditerranée. Ces flux s'inscrivent en effet dans la continuité des mouvements migratoires Sahel-Algérie et Sahel-Libye qui se sont organisés depuis le milieu du $\mathrm{XX}^{\mathrm{e}}$ siècle. Cette perspective historique bat ainsi en brèche toute impression de nouveauté ou de "renaissance» d'un Sahara marqué par la mobilité de ses populations. Celle-ci apparaît plutôt comme étant étroitement liée à l'histoire récente de la région, des indépendances (années 1950 et 1960) aux cycles de sécheresse du Sahel et aux guerres qui ont secoué la zone (années 1970 et 1980), et enfin aux différentiels de développement entre les versants nord-africain et sahélien du Sahara. Ce n'est qu'en prenant en compte cet arrière plan qu'il est possible d'envisager le Sahara à une échelle plus vaste, afin de saisir l'élargissement de l'espace migratoire qui s'est opéré depuis les années 1990 lorsqu'à la migration «à destination du Sahara » s'est ajoutée une «migration entre les deux rives du Sahara » [Bredeloup, Pliez, 2005] puis une « migration transsaharienne à destination de l'Europe ».

4 La lecture des cartes produites par l'Agence Frontex (http://www.frontex.europa.eu/newsroom/press_kit/), par des instituts comme l'ICMPD (International Centre for Migration Policy Development) ou encore régulièrement par la presse est de ce point de vue instructive. 


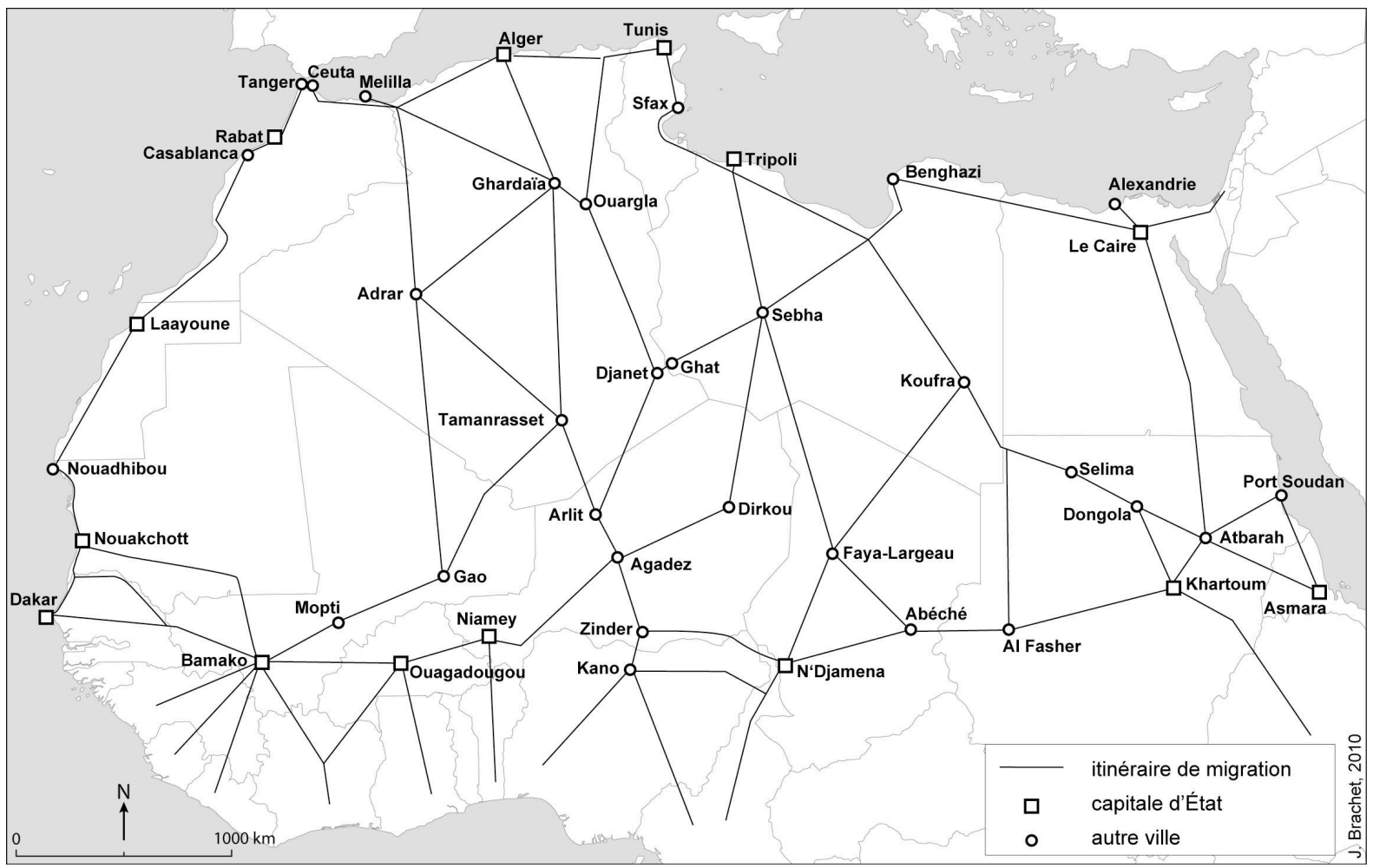

Carte : Principaux itinéraires des migrations (trans-)sahariennes

\section{LES MIGRATIONS AU SAHARA, UNE HISTOIRE INTRA-AFRICAINE DEJA ANCIENNE}

\section{Déséquilibres de peuplement et réseaux migratoires}

Faiblement peuplé en dépit d'une croissance démographique récente ${ }^{5}$, ce vaste espace d'environ 8 millions de $\mathrm{km}^{2}$ se caractérise aujourd'hui par un processus d'urbanisation qui frappe par son ampleur, sa rapidité et sa sélectivité spatiale. Outre le fait que la grande majorité des Sahariens vivent dorénavant dans des agglomérations et que la moitié d'entre eux vit le long des marges du désert, cette croissance démographique, inégale, contribue à différencier les versants maghrébin au Nord et sahélien au Sud ${ }^{6}$. Le déséquilibre des hiérarchies urbaines est en effet systématique (plus de 90 villes dans le Sahara maghrébin contre à peine une trentaine dans le Sahara sahélien [Bisson, 2003 : 108]) et renvoie à l'inégale intervention des États. En Afrique du Nord, du Maroc à l'Égypte, l'État s'est souvent fait démiurge, bitumant les pistes, désenclavant les oasis isolées, créant des pôles administratifs fonctionnels et des villes, purs produits des politiques publiques d'aménagement des années 1960 aux années 1980 [Cote, 2002; Pliez, 2011]. Ces villes se distinguent fortement des villes sahariennes anciennes, carrefours hérités d'une histoire séculaire d'échanges sahariens et transsahariens. À l'inverse, les États ont été beaucoup moins interventionnistes côté sahélien, faute de capacité financière le permettant.

Ce déséquilibre est l'un des moteurs des flux migratoires du Sud vers le Nord à partir des années 1950. Dès cette époque, des circulations migratoires se mettent progressivement en place entre le

5 La population saharienne, dans les limites du «Sahara français » (c'est-à-dire l'ensemble sous domination coloniale française comprenant le Maroc, l'Algérie, la Tunisie, la Mauritanie, le Mali, le Niger et le Tchad actuels, soit une superficie de 4,3 millions de $\mathrm{km}^{2}$ ), était estimée à 1,7 millions d'habitants en 1948, 2 millions en 1966 et 10 millions au milieu des années 1990.

6 Si la plupart des régions sahariennes gagnent des habitants, les régions méridionales de Libye et d'Algérie connaissent quant à elles des taux de croissance démographique particulièrement élevés, supérieurs à $4 \%$ par an. 
Sahel où l'inactivité, notamment agricole en saison sèche, touche une population jeune relativement nombreuse, et les régions sahariennes des États d'Afrique du Nord. Dans un premier temps, des travailleurs originaires du Sahel se rendent dans le Sahara algérien pour participer à la construction de bases militaires françaises destinées à accueillir plusieurs milliers d'hommes (à Reggane et In Ekker) a entraîné la venue de travailleurs peu qualifiés originaires du Sahel. Puis, au cours des années 1960, les dirigeants de l'Algérie nouvellement indépendante (1962) et de la Libye aux moyens décuplés grâce à la découverte et à l'exploitation de gisements de pétrole (à partir de 1961), vont mettre en place des politiques de développement des régions sahariennes alors que le déficit en main-d'œuvre est très important. La demande de transport croit et des camions font alors la navette entre certaines régions du Sahel et du Sahara, par exemple depuis le Darfour et le Nord du Tchad [Capot Rey, 1961] pour acheminer les hommes qui viennent travailler en Libye.

Les appels à la venue de travailleurs immigrés participent de manière déterminante au développement, au peuplement et à l'urbanisation du Sahara maghrébin [Bisson, 2003 ; Blin, 1990]. Il en va de même en Mauritanie où les gisements de fer situés dans le Nord du pays, découverts et exploités par les Français depuis 1952, attirent la main d'œuvre depuis le Sud plus densément peuplé [Bonte, 2000]. Le secteur de la pêche renforce l'attractivité de ce Nord saharien, notamment de Nouadhibou où des Sénégalais s'installent en nombre. À partir de 1957, la création et la construction de la nouvelle capitale Nouakchott offre également de multiples emplois pour les ressortissants des pays voisins (Sénégalais, Maliens et Guinéens en particulier), alors mieux formés que les Mauritaniens pour $70 \%$ encore nomades. Dans tous les cas de figures, les projets de développement économiques et les grands travaux qui s'ensuivent créent une forte demande de main d'œuvre peu qualifiée.

C'est dans ce contexte économique que survient la période de sécheresse qui touche le Sahel de 1969 à 1973, engendrant une famine et une grave crise agricole et pastorale qui secoue toute la région. Cette sécheresse fonctionne tantôt comme un accélérateur, tantôt comme un déclencheur des migrations à destination du Sahara algérien et libyen [Bernus, 1999]. L'espace migratoire se densifie entre le Sahel et les chantiers du Sahara central, puis s'étend progressivement de la Mauritanie au Soudan. Un nombre croissant de camions acheminent alors des pasteurs dont les troupeaux ont été décimés et qui viennent gagner de quoi le reconstituer ou se reconvertir dans d'autres activités, notamment le commerce.

Ces migrations deviennent bien souvent une source de revenu complémentaire notoire pour les familles des migrants. Ces derniers, essentiellement des hommes, jeunes, envoient en effet des mandats pendant leur absence ou rapportent des marchandises (biens manufacturés et produits alimentaires notamment) lors de leurs retours temporaires ou définitifs. Les flux sont d'ampleur puisqu'on estime qu'ils concernent la moitié des chefs de famille du nord du lac Tchad entre 1972 et 1979 [Clanet, 1981], ou encore un foyer sur cinq au Darfour [Couteaudier, 2005]. Dans une ville comme Agadez, au Niger, le passage de migrants crée des vocations et devient une source notoire de revenu pour les locaux les plus entreprenants. Des caravaniers touaregs ou toubous s'improvisent guides et passeurs de frontières, des transporteurs marchands du Sahel et du Maghreb se font également convoyeurs de migrants. Outre le secteur des transports, le logement et l'emploi de migrants de passage participent également de l'apparition et du développement de toute une économie du transit migratoire, dans des villes habituées à vivre de la circulation et des échanges marchands au long cours [Brachet, 2009].

L'information selon laquelle il est possible d'aller " tenter sa chance » en Mauritanie, en Algérie ou en Libye va progressivement se diffuser à travers toute l'Afrique de l'Ouest et séduire des individus aux motivations diverses, économiques mais aussi sociales ou politiques. Le rôle des États est alors déterminant, notamment avec la signature d'accords de libre circulation dans des cadres bilatéraux ou multilatéraux. Des réseaux de transport puis des réseaux migratoires intégrant des acteurs plus nombreux aux rôles complémentaires vont ainsi peu à peu se développer, structurant des itinéraires entre le Sahel et diverses régions du Maghreb. 


\section{Là où les transports passent, des routes se créent et des individus circulent}

Ce n'est pas sans raisons qu'un parallèle est parfois établi entre les routes marchandes transsahariennes d'hier et les routes migratoires d'aujourd'hui ${ }^{7}$. Celui-ci n'a pourtant rien de systématique. L'apparence d'échanges transsahariens « fluides » structurés le long de routes entre les deux rives du Sahara, cache en effet des associations de segments de parcours dont l'agencement et le contrôle relèvent d'allégeances, d'alliances et de complémentarités économiques. Et celles-ci ne valent que le temps d'un équilibre souvent précaire entre les groupes qui la jalonne, en anime la circulation et en capte la manne.

La colonisation, à partir de l'arrivée des Français en Algérie en 1830, et la concurrence des voies maritimes depuis le golfe de Guinée, vont entraîner une rupture des structures méridiennes d'échange antérieures. Les routes forgées sur l'association entre commerce et Islam sont démantelées et les diverses puissances coloniales réorganisent les territoires dominés dans des ensembles aux contours différents (Afrique française du Nord, AOF, AEF, Libye italienne et Vallée du Nil sous domination britannique). Les agencements qui sont alors opérés demeurent encore très prégnants dans la géopolitique régionale, par exemple autour des grandes routes qui convergent vers Sebha et Koufra, deux carrefours migratoires majeurs.

Dans les années 1830, les alliances établies entre la tribu des Awlad Sliman [Cordell, 1985] avec les Magarha, les Warfalla et les Guedadfa (tribu de Mouammar Kadhafi) leur permettent de contrôler une vaste région qui s'étend du nord du Lac Tchad à Syrte via le Fezzan. Leur puissance décline à la fin du XIX ${ }^{\mathrm{e}}$ siècle lorsque l'étau colonial se resserre. Marquée par l'aura religieuse et mystique de son fondateur, Mohamed Ali es-Senoussi, la confrérie Senoussiya construit alors une sorte de « multinationale » [Triaud, 1996] qui connaît une activité économique sans précédent sur les pistes entre Abéché et Benghazi jusqu'au début du $\mathrm{XX}^{\mathrm{e}}$ siècle.

La route des Awlad Sliman et celle de la Senoussiya se rappellent depuis régulièrement dans l'actualité de la région, du Niger au Soudan. Ce fut le cas lors de la longue guerre entre le Tchad et la Libye (1973-1994), puis lorsque Kadhafi a lancé sa politique africaine dans les années 1990, et enfin en 2011 à l'occasion du soulèvement contre son régime qui réveille les réseaux endormis de la Senoussiya. Plus au sud, le long des routes d'échange démantelées durant les décennies de domination coloniale, les Libyens disséminés au Tchad, au Niger mais aussi dans divers Etats d'Afrique centrale forment des communautés clés des échanges marchands transsahariens. Lorsque ces derniers reprennent avec vigueur dans les années 1990 [Bennafla, 2000], ils occuperont un rôle essentiel mais peu visible entre Afrique et Méditerranée ${ }^{8}$.

Au niveau de l'Algérie, les liens entre les populations arabophones algériennes et maliennes, qui ont permis aux commerçants algériens de dominer les échanges transfrontaliers dans cette région au $\mathrm{XX}^{\mathrm{e}}$ siècle, sont des liens commerciaux [Blin 1990] et familiaux anciens, qui se sont renforcés à partir des années 1970. De même, dans une ville comme Agadez la présence de grands commerçants arabes originaires d'Algérie est ancienne [Grégoire 1999] et des mouvements caravaniers ont mis en contact les populations touarègues d'Algérie et du Niger jusqu'à la fin des années 1960 [Bourgeot 1994]. Ces réseaux sociaux, familiaux et commerciaux ont été au cœur de la reprise des relations directes entre l'Afrique noire et le monde arabe à partir des années 1970, sous l'influence des États arabes rentiers de la région -Algérie, Irak, Libye et Arabie Saoudite notamment- [Schmitz, 2000].

Des routes renaissent également aux deux extrémités du Sahara. Entre Soudan, Érythrée, Éthiopie et Égypte, le Nil constitue toujours l'axe structurant de circulations nourries par les flux successifs de réfugiés d'Afrique de l'Est [Le Houérou, 2004] et plus récemment du Darfour. Ces circulations sont

\footnotetext{
7 Le terme « routes » désigne ici des axes de circulation souvent des pistes sableuses et non des infrastructures routières

8 À Koufra, en Libye, la signature des accords de paix avec le Tchad se traduit en 1994 par le départ des troupes qui vendent les camions militaires reconvertis par des opérateurs locaux dans le transport de marchandises, de bétail ou de migrants avec le Soudan et le Tchad.
} 
aujourd'hui renforcées par la construction d'une route qui rejoint le Sud de l'Égypte au Nord du Soudan. Dans l'espace ouest-saharien, du fait de la Guerre du Sahara Occidental, ces initiatives se dessinent plus tardivement. Il faut attendre la réouverture de la frontière entre le Maroc et la Mauritanie en 2002 et l'inauguration de la route goudronnée Nouakchott-Nouadhibou en 2004, dernier tronçon de la transsaharienne Tanger-Dakar, pour que les échanges commerciaux entre les espaces marocain, mauritanien et sénégalais, par ailleurs anciens, s'intensifient.

Ces flux rappellent également la forte intrication entre migration et religion [Bava, Picard, 2010]. Dans l'ouest-saharien, les réseaux religieux tissés par les membres des confréries de la Tijâniyya et de la Qadariyya perdurent grâce aux pèlerinages qui suscitent d'importants mouvements transfrontaliers de personnes et de biens [Ould Cheikh, 2004]. L'Islam constitue en soi un vecteur d'échange, que les étudiants africains boursiers entretiennent en se dirigeant vers les centres de formation en civilisation islamique de la Zitouna (Tunis), d'El Azhar (Le Caire), de la Da'wa Islamiya (Tripoli) ou de Fès au Maroc, mais aussi dans les universités francophones d'Algérie. Parallèlement, on constate une revitalisation des églises qui accueillent les migrants chrétiens dans toute l'Afrique du Nord.

Des itinéraires de circulation sont ainsi bien balisés, de longue date, entre les régions septentrionales et méridionales du Sahara, par des réseaux commerciaux, familiaux ou religieux animés tant par de grands commerçants arabes que par des populations de tradition nomade, Touaregs ou Toubou. La fluidité des échanges transfrontaliers n'en demeure pas moins pour partie tributaire des relations géopolitiques de voisinage. Lorsque les politiques africaines des États d'Afrique du Nord prennent de l'ampleur à partir des années 1980, l'appel à la migration atteint des dimensions continentales. Les routes nouvellement asphaltées et les villes récemment créées par les aménageurs, à la demande d'États volontaristes voulant la renaissance du Sahara, du Maroc à l'Égypte, deviennent les pivots de nouvelles circulations transsahariennes. Les mouvements migratoires qui se développent dans la seconde moitié du $\mathrm{XX}^{\mathrm{e}}$ siècle peuvent ainsi se greffer sur ces réseaux de circulation déjà existants, permettant leur essor rapide sur certains axes à partir des années 1990.

\section{Carrefours, étapes, relais : fabriques citadines le long des routes migratoires}

Vers le milieu des années 1990, on peut repérer l'émergence de villes de transit au croisement des principales routes vers lesquelles convergent les grands flux de circulation transsahariens. Les migrants d'Afrique occidentale et centrale, en empruntant les mêmes itinéraires que ceux utilisés par les migrants sahéliens depuis des décennies, participent de l'intensification des flux et de la croissance spectaculaire de ces villes-carrefours qui accueillent tour à tour des paysans quittant les oasis, des nomades, des réfugiés et des migrants. Sebha et Koufra (Libye), Tamanrasset (Algérie), Agadez (Niger) et Nouadhibou (Mauritanie) sont en conséquence à la fois des pôles administratifs régionaux mais aussi des carrefours cosmopolites, et comptent dorénavant plusieurs dizaines de milliers d'habitants'.

Des bourgades relais plus petites se sont multipliées aux points de jonction de routes secondaires ou aux points de rupture entre les routes asphaltées et les pistes. Ces petites agglomérations n'échappent pas à cette croissance soutenue, à commencer par tous les postes et villes frontières, notamment ceux d'Algérie et de Libye où furent installés des réfugiés du Sahara occidental, des Touaregs et des Toubous. À Dirkou (Niger), petite oasis devenue au cours des années 1990 un point nodal incontournable entre Agadez et le Sud libyen, le nombre de maisons a plus que doublé et la population, officiellement (sous-)estimée à 2000 habitants en 2001, oscillait trois ans plus tard entre 7000 et 10000 personnes [Brachet, 2005].

Les capitales des Etats riverains du Sahara, qui abritent des marchés nés du commerce qui s'est

9 Le Mali ouvre un consulat à Tamanrasset dès décembre 1982 en raison du nombre croissant de ses ressortissants dans cette ville. Pour des raisons similaires, renforcées par l'arrivée de nombreux agriculteurs et éleveurs ayant tout perdu pendant la sécheresse de 1983-1985 au Sahel, le Niger fait de même à Tamanrasset en 1985 [Grégoire, 1999], ainsi qu'à Sebha. Des consulats soudanais ouvrent également à Sebha et Koufra. 
développé dans le sillage des flux migratoires des années 1970 et 1980, constituent également des lieux importants de la migration transsaharienne. À Khartoum, le souk libya créé par les pasteurs du Darfour qui travaillent en Libye est devenu l'une des principales places commerçantes d'Afrique de l'Est [Drozdz, Pliez, 2005]. Des portions entières de la médina de Tripoli, à proximité du port, se sont transformées durant les années 1980 et 1990 en marché où se nouent les transactions entre commerçants arabes et africains.

Casablanca, Tunis, Tripoli ou Le Caire sont aujourd'hui labellisées «portes migratoires de l'Europe ». Cette appellation, sans fondement réel, apparaît de plus en plus éloignée d'une réalité qui change rapidement. Les portes sont closes et les étapes se transforment de plus en plus fréquemment en impasses où la durée du transit s'allonge et où les trajectoires se redéfinissent, par exemple vers Israël ou le Golfe depuis l'Égypte [Anteby-Yemini Lisa, 2008]. Lorsque des opportunités locales se dessinent, l'installation définitive est parfois envisageable.

La présence de migrants étrangers dans les villes sahariennes n'est en soit pas un phénomène nouveau, mais leur nombre est aujourd'hui plus important et leurs origines plus diverses [Boesen, Marfaing, 2007]. La question du rapport à l'étranger et de la perception de soi se pose alors avec une acuité nouvelle, ainsi que celle des relations sociales permises et produites par les villes. Au Sud du Sahara, dans les villes de transit où les migrants ne s'installent que de manière temporaire en attendant de poursuivre leur route, leur influence sur les sociabilités et les modes de vie des autres populations urbaines semble relativement limitée. Et c'est davantage au sein des collectifs de migrants qui se créent dès l'orée du désert que l'on assiste à l'émergence de relations nouvelles, de solidarités ou de devoirs entre des individus ayant le sentiment de partager pour partie un même destin et plus encore une même condition présente [Brachet, 2009]. En revanche, dans les villes où les migrants s'installent, volontairement ou par défaut, telles que Nouadhibou, Tamanrasset ou Sebha, leur présence influe davantage sur les modes de vie des populations locales et entraîne parfois, en parallèle, des tensions. Que ce soit en Afrique du Nord ou en Mauritanie [Choplin, Lombard, 2008 ; Streiff-Fénart, Poutignat, 2008], les ressortissants d'Afrique subsaharienne sont fréquemment désignés comme source de tous les maux, notamment dans les médias. Sur fond de racisme et de xénophobie, les étrangers noirs sont épisodiquement victimes de poussées de violence, comme ce fut par exemple le cas à Oran en 2005 ou de manière extrême à Tripoli en 2000 et 2010. En parallèle, de la Mauritanie à l'Égypte, les migrants s'organisent en collectifs pour se défendre et faire entendre leurs voix et leurs droits, soutenus par de nouvelles associations issues des « sociétés civiles » locales, notamment de défense des droits de l'homme [Alioua, 2009].

\section{LA POLITISATION D'UN PHENOMENE : LES MIGRATIONS AU SAHARA VUES D'EUROPE}

\section{Accroissement des flux et extension aux deux rives du Sahara}

Le fait que les migrants sahéliens reviennent d'Afrique du Nord en possession d'un pécule et de biens de consommation a largement contribué à la construction et à la diffusion de l'image d'une Libye et d'une Algérie prospères au sud du Sahara. Le poids des politiques sur la mobilité des individus n'en demeure pas moins important.

Face à l'échec de sa politique pan-arabe, et afin de limiter les effets de l'isolement de la Libye sur la scène internationale consécutif à l'embargo décreté contre ce pays par l'ONU en avril $1992^{10}$, Mouammar Kadhafi se lance dans une politique de rapprochement avec différents États africains et se prononce ouvertement en faveur d'une immigration africaine dans son pays. Ce changement d'orientation de la politique africaine de la Libye ${ }^{11}$ se traduira notamment par la signature d'accords de libre circulation dans des cadres bilatéraux ou multilatéraux, puis par la création en 1998 de la Cen-Sad (Communauté des États Sahélo-sahariens), et suscitera l'engouement de nombreux jeunes

$10 \quad$ Cet embargo sera suspendu en avril 1999.

11 Déjà amorcé avec la signature en juillet 1990 d'un accord de libre circulation des personnes avec le Soudan. 
africains aux motivations diverses.

À ce contexte géopolitique libyen s'ajoute notamment la dévaluation du franc CFA en janvier 1994, qui entraîne l'appauvrissement de toute une partie des «classes moyennes » des pays de la zone franc. Peu après, la mise en application des accords de Schengen en 1995 se traduit par la généralisation des régimes de visas en Europe et la complexification des conditions de leur délivrance pour les ressortissants africains. Ces facteurs économiques et politiques, sans être déterminants, ont également participé de l'émergence à petite échelle d'un contexte favorable à l'amplification des migrations entre les deux rives du Sahara et à la diversification des origines sociales et géographiques des migrants (féminisation, hausse du niveau d'instruction).

Parmi les migrants qui vont ainsi circuler en plus grand nombre en Afrique du Nord, une petite partie va se greffer sur les réseaux maghrébins de migration à destination de l'Europe, actifs là où des "morceaux d'Europe" sont proches (Îles Canaries, Gibraltar, Ceuta et Mellila, Lampedusa, Malte). Ces migrants demeurent peu nombreux, tant par rapport à l'ensemble des flux d'immigration à destination de l'Europe qu'au regard des migrations intra-africaines [Lessault, Beauchemin, 2009], mais font pourtant l'objet d'une mobilisation médiatique et politique impressionnante. La question de la gestion des flux migratoires entre ainsi en force dans le débat politique euro-africain.

\section{Le Sahara (in)contrôlé ou la nouvelle frontière migratoire de l'Europe}

La question des migrations transsahariennes à destination de l'Europe est de plus en plus politisée et médiatisée, alors même qu'elle est mal renseignée au plan quantitatif. L'hypothèse haute de centaines de milliers, voire de millions de personnes brandie régulièrement par les droites populistes européennes n'est d'évidence pas sérieuse. Lorsque Mouammar Kadhafi la relaie ou l'amplifie, c'est dans le cadre de négociations plus générales portant tantôt sur la sortie d'embargo de la Libye, tantôt sur le montant des dédommagements liés à la colonisation italienne [Bredeloup, Pliez, 2011]. Les ordres de grandeur donnés par l'agence Frontex ${ }^{12}$ conduisent à considérer qu'ils n'ont jamais excédé quelques dizaines de milliers de personnes par an. Les discours politiques n'en ont pas moins construit la nécessité de contrôler ces flux, supposée répondre à deux objectifs : protéger l'Union européenne et lutter contre le terrorisme.

Aussi, entre 2000 et 2010, les frontières méridionales de l'UE se sont démultipliées pour s'externaliser et descendre progressivement des côtes de la Méditerranée vers les côtes atlantiques de l'Afrique et le Sahara. Depuis la fin des années 1990, la lutte contre la migration fait l'objet d'une gestion «concertée » des flux migratoires entre l'Afrique et l'Europe. Pour cela, le partenariat Euro-méditerranéen, et en son sein le «groupe $5+5$ », invite au " dialogue » pays de l'Union du Maghreb Arabe (Maroc, Algérie, Libye, Mauritanie et Tunisie) et pays européens (Espagne, France, Italie, Malte et Portugal) ${ }^{13}$. Progressivement, l'UE soumet les États maghrébins, puis saharo-sahéliens, au jeu de ses incitations-pressions afin de passer des accords de réadmission et de coopération en matière lutte contre les migrations irrégulières, permettant de refouler les migrants qui auraient réussi à atteindre l'Europe. Les signatures de ces accords, qui se font parfois dans le cadre du FED (Fonds Européen de Développement) ${ }^{14}$, sont généralement assorties d'échanges commerciaux et de programmes dits de « coopération au développement ».

Les contrôles de l'UE sont également très importants et visibles le long de la côte atlantique.

12 L'Agence européenne pour la gestion de la coopération opérationnelle aux frontières extérieures des États membres de l'Union européenne, appelée Frontex, est une sorte de corps européen de gardes frontières. En Méditerranée et en Atlantique, Frontex organise des patrouilles de surveillance des côtes africaines, conjointement avec des agents des pays tiers concernés, afin d'intercepter en mer et de refouler les migrants supposés être en partance pour l'Europe.

13 Le «groupe $5+5$ » est une enceinte de dialogue politique informel réunissant des ministres des Affaires étrangères et parfois des ministres de l'Intérieur. Ses activités visent à consolider le processus de Barcelone, notamment sur la question des migrations Afrique-Europe.

14 Dans le cadre du $10^{\mathrm{e}}$ FED (2008-2013), 8 millions d'euros ont été débloqués pour la Mauritanie, et autant pour le Mali, afin d'aider ces pays à « mieux gérer les flux migratoires » [Migreurop, 2010]. 
L'agence Frontex surveille désormais les littoraux marocains, mauritaniens et sénégalais dans le cadre des programmes Héra qui, de 2006 à 2010, ont été financés à hauteur de 25 millions d'euros [Migreurop, 2010]. Les départs de migrants vers les îles Canaries se sont un temps déplacés de plus en plus au sud, jusqu'en Guinée, occasionnant des trajectoires toujours plus périlleuses. En parallèle, plusieurs grands programmes européens de lutte contre les migrations irrégulières sont mis en place au niveau des frontières méridionales de l'Algérie et de la Libye, utilisant des moyens similaires à ceux de l'agence Frontex. Cette démultiplication des lieux de contrôle et leur soustraitance par les pays de la zone saharienne ont rapidement eut des effets côté canarien : 2300 arrivées sont comptabilisées en 2009 contre 32000 en 2006, selon les chiffres du ministère de l'intérieur espagno ${ }^{15}$. Ces chiffres occultent des centaines de morts et des milliers d'arrestations, de refoulements et d'expulsions [Migreurop 2010].

À la sur-médiatisation des flux migratoires à destination de l'Europe, et à leur surestimation persistante dans nombre de discours politiques, s'ajoute depuis peu la question de la «menace terroriste » au Sahara. Les amalgames entre « terrorisme » et « migration clandestine » sont de plus en plus récurrents, la thématique s'invitant régulièrement dans les débats politiques au-delà des seuls États-Unis, dont l'administration Bush a lancé une guerre contre le terrorisme au Sahel dès 2003 [Keenan 2005; Lecocq, Schrijver, 2007]. Les initiatives algériennes de contrôle des circulations transfrontalières dans les régions désertiques [Bensaâd, 2009] sont aussi soutenues par les États-Unis. Ces derniers, dans une logique de lutte contre le terrorisme international et spécifiquement contre certaines organisations armées circulant dans cette partie du Sahara - tel le Groupe Salafiste pour la Prédication et le Combat, devenu AQMI en 2007 -, participent de la même dynamique de renforcement du contrôle des déplacements dans cette zone. L'Europe fait de même en aidant financièrement les gouvernements de la région qui se disent prêts à lutter contre le terrorisme, le trafic de drogue et les migrations irrégulières. Il en va ainsi en Mauritanie où, depuis l'été 2010, une dizaine de postes de contrôle supplémentaires ont été créés le long des frontières sénégalaise et malienne pour mieux contrôler les circulations. Une vingtaine de nouvelles brigades mobiles de gendarmes et des groupements spéciaux d'intervention antiterroristes ont pareillement été déployés. Cette surveillance accrue, qui se généralise dans tout le Sahara, se couple à une technicisation croissante des contrôles. Nombreux sont désormais les postes frontières sahéliens et sahariens à être équipés, ou en cours d'équipement, en matériel informatique permettant la détection de faux papiers et la création d'un fichier des étrangers avec prise d'empreintes biométriques. Le 10 juillet 2010, le gouvernement mauritanien a conlu un accord avec le groupe français Safran pour que l'état civil soit dorénavant géré par un système d'identification biométrique. Pour justifier le prix du contrat (18 millions d'euros), le ministre de l'Intérieur a invoqué la nécessité de passer au biométrique afin de "lutter contre la falsification des documents d'identification » et «contre l'immigration clandestine $»$.

À l'échelle régionale, il est question de mettre en place un visa unique biométrique pour les ressortissants de la CEDEAO. Programmé pour 2012, cet «Ecovisa», calqué sur le modèle du « visa Schengen», a pour finalité officielle de fluidifier les circulations entre les États de la Communauté et de mieux contrôler les entrées et sorties de l'espace communautaire. Dans les faits, la volonté des pouvoirs publics européens qui soutiennent ce projet n'est pas à dissocier de leur volonté de limiter les envies et possibilités de sortie des ressortissants de la zone vers l'Europe. Si l'on peut s'interroger sur la finalité (et la faisabilité) de ces projets biométriques, il est néanmoins évident que cette injonction sécuritaire perturbe les circulations de population à l'intérieur du continent.

Ce nouvel ordre géopolitique, qui repose sur une technicisation croissante des contrôles - radars, informatisation, biométrie - [Bigo, 2007], est présentée comme indispensable dans la lutte contre le terrorisme, ce qui permet d'éviter les critiques croissantes des associations internationales de

15 Ministère espagnol de l'Intérieur, Balance de la lucha contra la inmigración ilegal, 2009 :

www.mir.es/DGRIS/Balances/Balance_2009/pdf/np011502_bal_lucha_inm_ilegal_2009.pdf 
défense des droits de l'homme (Fédération Internationale pour la défense des Droits de l'Homme, Human Rights Watch, etc.) ou de celles, nombreuses mais moins influentes, qui ont vu le jour ces dernières années dans divers pays d'Afrique du Nord et de l'Ouest. Nouvelle entrave aux déplacements, cette technicisation touche directement les migrants qui sont de plus en plus appréhendés comme de potentiels clandestins, voire comme d'éventuels collaborateurs de groupes terroristes.

\section{Nouvelle géopolitique saharienne à l'aune des migrations : un espace bipolaire?}

Suite aux contrôles accrus des frontières maritimes de l'UE, étendus à la zone saharienne, le transit migratoire est globalement ralenti [Brachet, 2011] et laisse parfois place à des situations que l'on peut qualifier de «post-transit» [Choplin, 2010]. Dans ce nouveau contexte, la géopolitique de l'espace saharien se redéfinit et trois frontières se dessinent [Pliez, 2011]. La première correspond aux limites politiques de l'espace Schengen; elle est de plus en plus efficacement fermée. La deuxième, le long des côtes d'Afrique du Nord et de l'Ouest, prend la forme d'un espace-tampon, parsemé de camps de rétention et dans lequel s'opérerait le filtrage des migrants et des réfugiés [Clochard, Rekacewicz, 2006]. Une troisième frontière, aux contours flous et mouvants, quasi dématérialisée, se met en place par répercussion à des milliers de kilomètres au sud. Cette dernière frontière n'est pas une ligne mais un vaste espace saharo-sahélien où les circulations vers le nord sont de plus en plus entravées. En son sein, le long des routes, les migrants sont soumis à des contrôles, des taxes, des arrestations et des refoulements.

La gouvernance migratoire concourt alors à différencier les territoires et à dessiner un espace bipolaire. De "bons espaces », où se gère institutionnellement la migration, s'opposeraient à de « mauvais espaces», stigmatisés car assimilés aux «migrations clandestines ». Ces « mauvais espaces » peuvent correspondre à des morceaux de ville comme les quartiers de migrants dans les villes-carrefours de Tamanrasset, Agadez, Gao ou à des villes frontalières de transit comme Nouadhibou, Nioro-du-Sahel, Tinzaouten ou Dirkou. Il peut encore s'agir de zones d'interpellation (le long des côtes) ou de refoulement : par exemple, à la frontière Mali-Mauritanie le CIGEM $^{16}$ se charge de « recueillir» les migrants refoulés de Mauritanie ; à la frontière LibyeNiger, l'OIM s'occupe du retour des migrants installés en Libye autant que de l'acheminement vers le sud de ceux qui en sont expulsés ou qui en fuient les conflits depuis février 2011. Ces mauvais espaces peuvent également prendre la forme de points ou de lignes : camps et autres centres de rétention ; poste de secours de la Croix Rouge espagnole; poste de police, de gendarmerie, de l'armée ou des douanes le long des pistes, routes et frontières ; murs et grillages qui ceinturent des enclaves comme Ceuta ou les camps... Les noms mêmes attribués à ces lieux rendent compte de la stigmatisation dont les migrants sont victimes : ghetto (lieux de restauration et d'hébergement situés dans les villes traversées), Kandahar (frontière Maroc/Mauritanie), Guantanamito (nom donné au centre de rétention de Nouadhibou), le Bouchon (checkpoint à l'entrée de Nouadhibou) ${ }^{17}$, Tchad (zone d'attente pour l'embauche journalière) et cailloux-ville (refuge nocturne en sortie de ville) à Tamanrasset. A ces espaces où sont susceptibles de passer, de s'installer et tout simplement de vivre les migrants, s'opposent les espaces où se «gère » la question migratoire. Si les premiers, associés aux circulations irrégulières, sont généralement situés dans les périphéries des territoires nationaux ou les périphéries urbaines, le « problème » est quant à lui pris en charge depuis les centres urbains et par des acteurs extérieurs. Dit autrement, ce sont dans les quartiers centraux et d'affaires de Nouakchott, Bamako, Tripoli, Niamey, Dakar ou encore Rabat qu'ont été installés les antennes du

16 Le CIGEM (Centre d'information et de gestion des migrations) a été inauguré en octobre 2008 à Bamako. Il est présenté comme un espace de dialogue ouvert entre le Mali et l'Union européenne, en vue d'élaborer une approche concertée des questions migratoires. En réalité, ce centre est avant tout destiné à lutter contre l'immigration irrégulière et à promouvoir les migrations de travail légales.

17 Le lieu-dit «Le Bouchon » constitue un point de passage obligatoire pour qui veut pénétrer sur la presqu'île de Nouadhibou. Il se situe précisément à l'endroit où il n'existe qu'une infime bande de terre entre la mer, le chemin de fer et la frontière du Sahara Occidental. 
HCR, de l'OIM et des Délégations européennes, qu'ont ouvert les bureaux d'experts chèrement payés et les sièges d'ONG censées " améliorer les conditions de vie des populations migrantes », que se dressent les églises qui « volent au secours » des migrants. L'argent des bailleurs est arrivé et a été investi dans des centres urbains, bien souvent situés en dehors de l'espace saharien proprement dit. Cette géopolitique bipolaire n'est nullement figée dans le temps et l'espace, «bons » et « mauvais » espaces peuvent être contigus (par exemple à l'intérieur d'une ville), et un même lieu peut se retrouver tour à tour dans une catégorie puis dans l'autre.

Un parallèle peut être établi entre cette dichotomie spatiale et les catégories de "bons» et «mauvais » migrants, élaborées en Europe et reprises en Afrique. Interlocuteurs privilégiés des bailleurs de fonds, les migrants installés depuis longtemps se réapproprient la rhétorique de la clandestinité développée en Europe. Ils contribuent à figer les catégories, étant entendu qu'eux appartiennent à la catégorie des «bons migrants", en situation régulière, généralement entrepreneurs transnationaux. Ils cherchent ainsi à se distinguer des migrants en transit, ceux qu'ils appellent parfois, non sans un certain mépris, les «aventuriers » et qui sont perçus comme liés à toutes sortes d'activités et de comportements dépréciés voire illicites (drogue, alcool, prostitution). En ce sens, une nouvelle géographie de la gouvernance migratoire s'est progressivement dessinée, opposant de façon peu nuancée différents espaces : les centres villes des capitales sahéliennes ou maghrébines où se gère la migration $v s$. les périphéries/marges sahariennes des États, où seraient lesdits « clandestins », passeurs, centres de rétention...

\section{Conclusion}

Au début de l'année 2010, les effets du contrôle accru des frontières conduisaient l'agence Frontex à considérer que la rive Sud de la Méditerranée n'était plus la voie d'entrée majeure des migrants irréguliers vers l'Europe. Les apparentes frontières migratoires de l'UE se déplacent vers l'est ${ }^{18}$, aussi vite que les caméras qui braquent désormais leurs objectifs sur Istanbul, Tel-Aviv ou Sofia, ces métropoles labellisées à leur tour «nouvelles » portes d'entrées de l'Europe.

À l'heure où l'on annonçait que « la mer se ferme » au large de la Mauritanie [Choplin, 2010], du Maroc, de la Tunisie et de la Libye [Bredeloup, Pliez, 2011], le " Printemps arabe » a réactivé les positions xénophobes et sécuritaires qui agitent de nouveau le spectre de l'invasion migratoire, trahissant ainsi la certitude avancée derrière le paravent technique : les frontières sont contrôlées, les coopérations bilatérales fonctionnent, et pourtant, la Méditerranée s'ouvrirait de nouveau. À écouter nombre de discours politiques, particulièrement en France et en Italie, le péril migratoire en provenance du Sud et la menace terroriste, embarqués sur une même pirogue, seraient une fois de plus aux portes de l'Europe.

Le décalage entre les réalités des mobilités sahélo-sahariennes et les politiques migratoires euroméditerranéennes demeure une constante. Des questions essentielles restent pourtant sans réponses au-delà des trajectoires éphémères de ce qui ne semblait être qu'un épiphénomène : le transit vers l'Europe. Que reste-t-il au Sahara une fois que les médias et les politiques ont déplacé leurs regards ? Que reste-t-il de ces flux sahariens, de ces villes qui ponctuent les routes, de ces activités qui, un temps, ont été dynamisées par ces mouvements de population ? Le Sahara est non seulement traversé, mais aussi travaillé par ces migrants qui y séjournent ou s'y installent depuis plusieurs décennies. Ils s'inscrivent dans des systèmes migratoires régionaux encore peu connus et pourtant largement perturbés par la focalisation des regards sur les frontières de l'UE et l'externalisation de ses contrôles. Ils sont des acteurs essentiels de la fabrique d'une géographie que l'on a toujours autant de peine à saisir, celle du Sahara (im)mobile. 


\section{Bibliographie}

AliouA M. (2009), "Le "passage au politique" des transmigrants subsahariens au Maroc », in A. Bensaâd (ed.), Le Maghreb à l'épreuve des migrations subsahariennes. Immigration sur émigration, Paris: Karthala, p. 281-306.

AnteBy-Yemini L. (2008), « Migrations africaines et nouveaux enjeux de la frontière israélo-égyptienne », Cultures \& Conflits, (72), p. 77-99.

BAVA S., PICARD J. (2010), "Les nouvelles figures religieuses de la migration africaine au Caire" in Migrations et transformations des paysages religieux, $\mathrm{n}^{\circ}$ 56, Autrepart (56), p. 153-170.

BENNAFLA K. (2000), "Tchad : l'appel des sirènes arabo-islamiques", Autrepart (16), p. 67-86.

BENSAÂD A. (2009), "L'immigration en Algérie. Une réalité prégnante et son occultation officielle", in A. Bensaâd (ed.), Le Maghreb à l'épreuve des migrations subsahariennes. Immigration sur émigration, Paris: Karthala, p.15-42.

BERNUS E. (1999), "Exodes tous azimuts en zone sahélo-saharienne", in V. Lassailly-Jacob, J.-Y. Marchal, A. Quesnel (eds), Déplacés et réfugiés. La mobilité sous contrainte, Paris: Éditions de l'IRD, p. 195208.

BIGO D. (2007), "Detention of foreigner, States of exception, and the social practices of control of the Banopticon" in Rajaram P. K., Grundy-Warr C. (eds), Borderscapes: Hidden Geographies and Politics at Territory's Edge, Minneapolis : University of Minesotta Press, p. 5-49.

BISSON J. (2003), Mythes et réalités d'un désert convoité : le Sahara, Paris, L'Harmattan, 480 p.

BLIN L. (1990), L'Algérie du Sahara au Sahel, Paris: L'Harmattan, 501 p.

Boesen E., MARFAING L. (eds) (2007), Les nouveaux urbains dans l'espace Sahara-Sahel. Un cosmopolitisme par le bas. Paris: Karthala-ZMO, $332 \mathrm{p}$.

BONTE P. (2000), , « Faire fortune au Sahara : permanences et ruptures », Autrepart, 16, 2000, p. 49-65.

Bourgeot A. (1994), "L'agro-pastoralisme des Touaregs Kel Owey (Aïr)", in L. Bridel, A. Morel, I. Ousseini (eds) (eds), Au contact Sahara-Sahel : milieux et sociétés du Niger - volume I, Revue de Géographie Alpine, p. 137-155.

BRACHET J. (2005), "Migrants, transporteurs et agents de l'État : rencontre sur l'axe Agadez-Sebha", Autrepart (36), p. 43-62.

BRACHET J. (2009), Migrations transsahariennes. Vers un désert cosmopolite et morcelé (Niger), Paris: Éditions du Croquant, $322 \mathrm{p}$.

BRACHET J. (2011, à paraître), «Stuck in the Desert. Hampered Mobility among Transit Migrants in Northern Niger », in J. Streiff-Fénart and A. Wa Kabwé-Segatti (eds.), The challenge of the threshold. Border closure and migration movements in Africa, Lexington Books: Lanham, MD.

Bredeloup S., Pliez O. (2005), Migrations entre les deux rives du Sahara, Autrepart, n³6, 199 p.

Bredeloup S., Pliez O. (2011), The Libyan Migration Corridor, EU-US Immigration Systems, Robert Schuman Centre for Advanced Studies, San Domenico di Fiesole (FI): European University Institute.

CAPOT REY R. (1961), Borkou et Ounianga. Étude de géographie régionale, Alger. Institut de recherches sahariennes, $182 \mathrm{p}$.

Choplin A. (2010), "Quand la mer se ferme : du transit au post-transit migratoire en Mauritanie", Hommes et migrations, $\mathrm{n}^{\circ} 1286-87$, pp. 74-95

Choplin A., Lombard J., (2008), "Migrations et recompositions spatiales en Mauritanie. « Nouadhibou du monde ». Ville de transit... et après ?", Afrique contemporaine, 4 (228): 151-170.

Clanet J.C. (1981), "L'émigration temporaire des Toubou du Kanem vers la Libye», Cahiers géographiques de Rouen, (15), p.17-33.

ClOCHARD O., REKACEWICZ P. (2006), "Des morts par milliers aux portes de l'Europe", http://www.mondediplomatique.fr/cartes/mortsauxfrontieres

Cordell D.D., (1985), « The Awlad Sulayman of Libya and Chad: Power and Adaptation in the Sahara and Sahel », Canadian Journal of African Studies, 19 (3), pp. 319-43.

Cote M. (2002), Le Sahara, cette autre Méditerranée, Méditerranée, Aix, n³-4, 108 p.

COUTEAUdier T., et alii (2005), Markets, Livelihoods and Food Aid in Darfur: A Rapid Assessment and Programming Recommendations, EC-FAO-USAID, $70 \mathrm{p}$.

DrozDz M., PliEZ O. (2005), «Entre Libye et Soudan, la fermeture d'une piste transsaharienne », in Les migrations entre les deux rives du Sahara, Autrepart-Armand Colin, n³6, p. 63-80.

GRÉGOIRE E. (1999), Touaregs du Niger, le destin d'un mythe, Paris, Khartala, 339 p.

KEENAN J. (2005), "Waging War on Terror: The Implications of America's 'New Imperialism' for Saharan 
Peoples", The Journal of North African Studies, 10 (3): 619-647.

Le Houerou F. (2004), Passagers d'un monde à l'autre, migrants forcés éthiopiens et érythréens, Paris, L'Harmattan, $201 \mathrm{p}$.

LeCOCQ B., SCHRIJVER P. (2007), "The War on Terror in a haze of dust : potholes and pitfalls on the Saharan front", Journal of Contemporary African Studies, 25 (1): 141-166.

Lessault D., BEAUCHEMIN C. (2009), "Ni invasion, ni exode. Regards statistiques sur les migrations d'Afrique subsaharienne", Revue Européenne des Migrations Internationales, 25 (1): 163-194.

Mbembe A. (2000), "At the Edge of the World: Boundaries, Territoriality, and Sovereignty in Africa", Public Culture 12 (1) : 259-284. (traduction française «Les frontières mouvantes du continent africain », Le Monde Diplomatique, 11. 1999).

Migreurop (2010), Aux frontières de l'Europe. Contrôles, enfermements, expulsions. Rapport 2009-2010, Paris: Migreurop, 128.

Ould CHEIKH A. W. (2004), «Espace confrérique, espace étatique : le mouridisme, le confrérisme et la frontière mauritano-sénégalaise », in Z. OULD AHMED SALEM (éd.) Les trajectoires d'un Étatfrontière, Dakar, CODESRIA, p. 113-140.

PLIEZ O. (2011, à paraître), Les cités du désert. Des villes sahariennes aux saharatowns, PUM-IRD, Toulouse.

SCHMitz J. (2000), «L'islam en Afrique de l'Ouest : les méridiens et les parallèles », in Afrique noire et monde arabe, continuités et ruptures, Autrepart, Cahiers des Sciences Humaines, Ed de l'Aube, $\mathrm{n}^{\circ} 16$, $181 \mathrm{p}$.

SPIGA S. (2005), «Aménageurs et migrants subsahariens dans les villes du Grand Sud algérien », in Les migrations entre les deux rives du Sahara, Autrepart, Armand Colin - IRD, n³6, p.81-104.

Streiff-FÉnART J., POUTignat P. (2008), « Nouadhibou "ville de transit" ? Le rapport d'une ville à ses étrangers dans le contexte des politiques de contrôle des frontières de l'Europe », Revue Européenne des Migrations Internationales, 24 (2): 193-217.

TRIAUD J.-L. (1996), « Les "trous de mémoire" dans l'histoire africaine. La Sanûsiyya au Tchad : le cas du Ouaddaï », Revue Française d'Histoire d'Outre-Mer, vol. 83, n 311, $2^{\text {ème }}$ trimestre 1996 : 5-23. 